\title{
Erratum to: Novel diffusive gradients in thin films technique to assess labile sulfate in soil
}

\author{
Ondrej Hanousek $^{1,2} \cdot$ Sean Mason ${ }^{3} \cdot$ Jakob Santner $^{4}(D \cdot$ \\ Md Mobaroqul Ahsan Chowdhury ${ }^{3} \cdot$ Torsten W. Berger $^{2} \cdot$ Thomas Prohaska $^{1}$
}

Received: 10 November 2016 / Accepted: 10 November 2016 / Published online: 22 November 2016

(C) Springer-Verlag Berlin Heidelberg 2016

\section{Erratum to: Anal Bioanal Chem}

DOI 10.1007/s00216-016-9801-8

In the acknowledgements of the original publication the authors unfortunately failed to mention one body providing funding for this work. The complete acknowledgements read:

\section{Acknowledgments}

Open access funding was provided by the University of Natural Resources and Life Sciences Vienna (BOKU). This study was funded by the Austrian Science Fund (FWF): P23861-B16 and P27571-BBL. Additional funding was provided by the province of Lower Austria, which co-funds project P27571-BBL. Melanie Diesner is acknowledged for her support in the laboratory work, and Christoph Hoefer and Andreas Kreuzeder are acknowledged for their expert advice on the DGT technique.

The online version of the original article can be found at http://dx.doi. org/10.1007/s00216-016-9801-8.

Jakob Santner

jakob.santner@boku.ac.at

1 Department of Chemistry - VIRIS Laboratory, University of Natural Resources and Life Sciences Vienna, Konrad-Lorenz-Strasse 24, 3430 Tulln, Austria

2 Institute of Forest Ecology, University of Natural Resources and Life Sciences Vienna, Peter-Jordan-Strasse 82, 1190 Vienna, Austria

3 School of Agriculture, Food and Wine, University of Adelaide and the Waite Research Institute, Adelaide, South Australia 5064, Australia

4 Division of Agronomy, University of Natural Resources and Life Sciences, Vienna, Konrad-Lorenz-Strasse 24, 3430 Tulln, Austria 